PROCEEDINGS OF THE

AMERICAN MATHEMATICAL SOCIETY

Volume 128, Number 7 , Pages 1917-1926

S 0002-9939(00)05235-7

Article electronically published on February 25, 2000

\title{
BETTI NUMBERS OF MODULES OF ESSENTIALLY MONOMIAL TYPE
}

\author{
SHOU-TE CHANG
}

(Communicated by Wolmer V. Vasconcelos)

\begin{abstract}
Let $R$ be a Noetherian local ring. In this paper we supply formulae for computing the ranks of syzygy and Betti numbers of $R$-modules of essentially monomial type. These modules are defined with respect to various $R$-regular sequences. For example, finite length modules of monomial type over regular local rings of dimension $n$ are modules of essentially monomial type with respect to $R$-regular sequences of length $n$. If a module is of essentially monomial type with respect to an $R$-regular sequence of length $n$, then the rank of its $i$-th syzygy is at least $\left(\begin{array}{c}n-1 \\ i-1\end{array}\right)$ and its $i$-th Betti number is at least $\left(\begin{array}{c}n \\ i\end{array}\right)$.
\end{abstract}

\section{INTRODUCTION}

Throughout this paper the ring $(R, m, k)$ is always a Noetherian local ring.

Write $\beta_{i}^{R}(M)$, or $\beta_{i}(M)$ if $R$ is not emphasized, for the $i$-th Betti number of an $R$-module, and write $r_{i}^{R}(M)$, or $r_{i}(M)$ if $R$ is not emphasized, for the rank of the $i$-th syzygy of $M$. We will supply formulae for computing $r_{i}(M)$ and $\beta_{i}(M)$ of modules of essentially monomial type (see the Main Theorem). In fact we have $\beta_{i}(M)=r_{i}(M)+r_{i+1}(M)$.

Definition 0.1. Let $U=\left\{u_{1}, u_{2}, \ldots\right\}$ be an infinite set of indeterminates and let $\Lambda$ be the set of monomials in these indeterminates.

Let $\mathbf{u}$ be a sequence of $n$ distinct indeterminates taken from $U$. Let $f, g \in \Lambda$. We say $f \mathbf{u}$-divides $g$, denoted $\left.f\right|_{\mathbf{u}} g$, if $g / f$ is a monomial solely in elements of $\mathbf{u}$. We use $\left.\left.f\right|_{\mathbf{u}} g\right|_{\mathbf{u}} h$ to denote the situation $f \mathbf{u}$-divides $g$ and $g \mathbf{u}$-divides $h$. A finite subset $T$ of $\Lambda$ is called a $\mathbf{u}$-segment if every $g \in \Lambda$ with $\left.\left.f\right|_{\mathbf{u}} g\right|_{\mathbf{u}} h$ for some $f, h \in T$ is in $T$.

To simplify notation we will assume that $\mathbf{u}=u_{1}, \ldots, u_{n}$. Suppose we are given a $\mathbf{u}$-segment $T$ and an $R$-regular sequence $\mathbf{x}=x_{1}, \ldots, x_{n}$. Let $|T|=l$ and let $F$ be the free $R$-module of rank $l$ with free generators $\left\{w_{f}\right\}_{f \in T}$. Let $N$ be the submodule of $F$ generated by

$$
\left\{x_{k} w_{f}-w_{u_{k} f}: u_{k} f \in T, f \in T\right\} \cup\left\{x_{k} w_{f}: u_{k} f \notin T, f \in T\right\} .
$$

Then $F / N$ is called the $R$-module associated with $T$ with respect to $(\mathbf{u}, \mathbf{x})$.

Received by the editors March 24, 1998 and, in revised form, September 1, 1998.

1991 Mathematics Subject Classification. Primary 13D25, 18G10; Secondary 13H05.

The author is partially supported by an N.S.C. grant of R.O.C.

(C)2000 American Mathematical Society 
We say an $R$-module is of essentially monomial type with respect to an $R$-regular sequence $\mathbf{x}$ if it is isomorphic to a module associated with some $\mathbf{u}$-segment $T$ with respect to some $(\mathbf{u}, \mathbf{x})$. We say an $R$-module is of essentially monomial type if it is isomorphic to a direct sum of modules of essentially monomial type with respect to various $R$-regular sequences.

In $\S 2$ we will set up a free resolution for $M$, the module associated with some $\mathbf{u}$-segment $T$ with respect to some $(\mathbf{u}, \mathbf{x})$. After tensoring with $k$, the problem of finding $r_{i}(M)$ and $\beta_{i}(M)$ is reduced to calculating the ranks of certain matrices with entries in $k$, which are not as difficult as they look once one has some experience handling these matrices. For example, 4 is a good reference for studying these matrices in question. However, this task is greatly simplified by our Main Theorem below.

Theorem 0.2 (Main Theorem). Let $\mathbf{u}=u_{1}, \ldots, u_{n}$. Suppose we are given a local ring $R$, a u-segment $T$ and an $R$-regular sequence $\mathbf{x}=x_{1}, \ldots, x_{n}$. Let $M$ be the module associated with $T$ with respect to $(\mathbf{u}, \mathbf{x})$. Write $u_{1} T$ for the set $\left\{u_{1} f: f \in\right.$ $T\}$. Let $T^{\prime}=\left\{f \in T: u_{1} f \notin T\right\}$ and $T^{\prime \prime}=\left\{f \in T: f \notin u_{1} T\right\}$. Then $T^{\prime}$ and $T^{\prime}$ are $\overline{\mathbf{u}}$-segments where $\overline{\mathbf{u}}=u_{2}, \ldots, u_{n}$. Moreover, let $M^{\prime}$ and $M^{\prime \prime}$ be the modules associated with $T^{\prime}$ and $T^{\prime \prime}$ respectively with respect to $(\overline{\mathbf{u}}, \overline{\mathbf{x}})$ where $\overline{\mathbf{x}}=x_{2}, \ldots, x_{n}$. Then

$$
r_{i}(M)=r_{i-1}\left(M^{\prime}\right)+r_{i}\left(M^{\prime \prime}\right) .
$$

From this theorem the following corollary follows immediately.

Corollary 0.3. Let $M$ be a module of essentially monomial type with respect to an $R$-regular sequence of length $n$. Then $r_{i}(M) \geq\left(\begin{array}{c}n-1 \\ i-1\end{array}\right)$ and $\beta_{i}(M) \geq\left(\begin{array}{c}n \\ i\end{array}\right)$.

When $M$ is a finite length module over a regular local ring of dimension $n$, Horrocks' question asks that if $\beta_{i}(M)$ is at least $\left(\begin{array}{c}n \\ i\end{array}\right)$ and if $r_{i}(M)$ is at least $\left(\begin{array}{c}n-1 \\ i-1\end{array}\right)$. Both questions are known to be true up to dimension 4. These two questions are also open even if we drop the assumption that $R$ is regular local. (For more information, related problems and results see [4], 5], 6], [7], [8], [10], [11, [12, [13, [14, [15], etc.) In 10 Evans and Griffith gives a lower bound to the Betti numbers of finite length modules of monomial type which confirms Horrocks' questions for this special case. To be more precise, they consider direct sums of $R / I$ where $R=k\left[\left[x_{1}, \ldots, x_{n}\right]\right]$ is a polynomial ring of $n$ variables over a field $k$ and $I$ is generated by a set of monomials in $\mathbf{x}=x_{1}, \ldots, x_{n}$. They showed that $\beta_{i}(R / I) \geq\left(\begin{array}{c}n \\ i\end{array}\right)$. In $\S 1$ we will see that this is a special case of our results. Our approach has three advantages. Our approach is characteristic free. We give information about $r_{i}(M)$ as well. And instead of giving a lower bound we actually have formulae for computing $r_{i}(M)$ and $\beta_{i}(M)$. We will supply examples to familiarize readers with the notation and technique. For purpose of demonstration we will calculate $r_{i}\left(R / I^{t}\right)$ when $I$ is generated by an $R$-regular sequence in $\S 1$.

\section{EXAMPLES}

Example 1.1. Consider $R / I$ where $I$ is generated by monomials in an $R$-regular sequence $\mathbf{x}=x_{1}, \ldots, x_{n}$ such that $\sqrt{I}=\sqrt{\left(x_{1}, \ldots, x_{n}\right)}$. Finite length modules of monomial type that Evans and Griffith considered are such examples. We will demonstrate that these modules are of essentially monomial type with respect to $\mathbf{x}$. Let $\mathbf{u}=u_{1}, \ldots, u_{n}$. Let $T$ be the set of monomials $f(\mathbf{u})=f\left(u_{1}, \ldots, u_{n}\right)$ 
$=u_{1}^{\alpha_{1}} \cdots u_{n}^{\alpha_{n}}$ such that $f(\mathbf{x})=f\left(x_{1}, \ldots, x_{n}\right) \notin I$. The set $T$ is finite since $\left(x_{1}, \ldots, x_{n}\right)^{N} \subseteq I$ for some $N$. Furthermore, note that $T$ is a $\mathbf{u}$-segment. Let $f$ be such that $\left.\left.f\right|_{\mathbf{u}} g\right|_{\mathbf{u}} h$ for some $f, h \in T$. Then $g(\mathbf{x}) \notin I$ since $h(\mathbf{x}) \notin I$. Thus $g \in T$. In particular note that $1 \in T$.

Let $\left\{w_{f}\right\}_{f \in T}$ be the free generators of $F$. Let $\phi$ map $F$ onto $R / I$ by sending $w_{f}$ to the coset of $f(\mathbf{x})$. We want to identify the class of $w_{f}$ with the coset of the monomial $f(\mathbf{x})$. Note that $(0-1)$ precisely describes the relations between the monomials of $R$ not already in $I$. It is not hard to see that the submodule $N$ of $F$ generated by (0-1) is indeed the kernel of $\phi$. Obviously $N \subseteq \operatorname{Ker} \phi$. Now let $v=$ $\sum_{f \in T} a_{f} w_{f} \in \operatorname{Ker} \phi$. By a straightforward computation we have $w_{f}-f(\mathbf{x}) w_{1} \in N$ for each $f \in T$. Hence we have $v=v^{\prime}+\sum_{f \in T} a_{f} f(\mathbf{x}) w_{1}$ for some $v^{\prime} \in N$. Let $a=\sum_{f \in T} a_{f} f(\mathbf{x})$. Then $a \in I$. It suffices to show that $a w_{1} \in N$, find $f_{1}, \ldots, f_{s} \in T$ such that $I$ is generated by $x_{k_{1}} f_{1}(\mathbf{x}), \ldots, x_{k_{s}} f_{s}(\mathbf{x})$, and note that $x_{k_{t}} w_{f_{t}}$ are in the set $(0-1)$ for each $1 \leq t \leq s$. Let $a=\sum_{t=1}^{s} b_{t} x_{k_{t}} f_{t}(\mathbf{x})$ where $b_{t} \in R$. Then $a w_{1}=\sum_{t} b_{t} x_{k_{t}}\left(f_{t}(\mathbf{x}) w_{1}-w_{f_{t}}\right)+\sum_{t} b_{t} x_{k_{t}} w_{f_{t}} \in N$. Thus $R / I$ is isomorphic to the $R$-module associated with the $\mathbf{u}$-segment $T$ with respect to $\mathbf{x}$.

It is worth mentioning that submodules of $R / I$ generated by cosets of monomials in $\mathbf{x}$ are also of essentially monomial type. Suppose $L$ is such a submodule. Let $T^{\prime}=\{f \in T: f(\mathbf{x})+I \in L\}$. It is easy to see that $T^{\prime}$ is a $\mathbf{u}$-segment and that $L$ is isomorphic to the module associated with $T^{\prime}$ with respect to $(\mathbf{u}, \mathbf{x})$.

If $M_{1}$ and $M_{2}$ are the modules associated with the finite sets $T_{1}$ and $T_{2}$ respectively with respect to $(\mathbf{u}, \mathbf{x})$, find $u_{\alpha}$ and $u_{\beta}$ not already appearing in $T_{1} \cup T_{2}$. We now create a new $\mathbf{u}$-segment $T=\left\{u_{\alpha} f: f \in T_{1}\right\} \cup\left\{u_{\beta} g: g \in T_{2}\right\}$. It is easy to see that $T$ is a $\mathbf{u}$-segment and that the module associated with $T$ with respect to $(\mathbf{u}, \mathbf{x})$ is isomorphic to $M_{1} \oplus M_{2}$. Hence a finite direct sum of modules of essentially monomial type is of essentially monomial type.

Example 1.2. Let $x_{1}, \ldots, x_{n}$ be an $R$-regular sequence and let $I$ be the ideal generated by this regular sequence. We proceed to find $r_{i}\left(R / I^{t}\right)$.

Let $\mathbf{u}=u_{1}, \ldots, u_{n}$ and let $\mathbf{x}=x_{1}, \ldots, x_{n}$. Let $T$ be the set of monomials $f$ solely in $\mathbf{u}$ with $\operatorname{deg} f<t$. From the discussion in Example 1.1 we have that $R / I^{t}$ is isomorphic to the $R$-module associated with $T$ with respect to $(\mathbf{u}, \mathbf{x})$. Let $\overline{\mathbf{u}}=u_{2}, \ldots, u_{n}$ and let $\overline{\mathbf{x}}=x_{2}, \ldots, x_{n}$. Let $T^{\prime}=\left\{f \in T: u_{1} f \notin T\right\}=\{f \in T:$ $\operatorname{deg} f=t-1\}$ and let $T^{\prime \prime}$ be the set of the monomials $f$ solely in elements of $\overline{\mathbf{u}}$ with $\operatorname{deg} f<t$. Let $M^{\prime}$ and $M^{\prime \prime}$ be the modules associated with $T^{\prime}$ and $T^{\prime \prime}$ respectively with respect to $(\overline{\mathbf{u}}, \overline{\mathbf{x}})$. Let $J$ be generated by $x_{2}, \ldots, x_{n}$. We can immediately see that $M^{\prime \prime}$ is isomorphic to $R / J^{t}$. By definition,

$$
\begin{aligned}
M^{\prime} & \simeq \bigoplus_{\operatorname{deg} f=t-1} R w_{f} / \sum_{\substack{2 \leq k \leq n \\
\operatorname{deg} f=t-1}} R x_{k} w_{f} \\
& =\text { a direct sum of }\left(\begin{array}{c}
n+t-2 \\
t-1
\end{array}\right) \text { copies of } \frac{R}{J}
\end{aligned}
$$

Using Theorem 0.2 , when $i=1$ we have $r_{1}\left(R / I^{t}\right)=r_{1}\left(R / J^{t}\right)=1$ by induction on $n$. Now let $i \geq 2$. By Theorem 0.2 we have that $r_{i}\left(R / I^{t}\right)=\left(\begin{array}{c}n+t-2 \\ t-1\end{array}\right) r_{i-1}(R / J)+$ 
$r_{i}\left(R / J^{t}\right)$. Thus by induction on $n$ we have that

$$
\begin{aligned}
r_{i}\left(R / I^{t}\right) & =\left(\begin{array}{c}
n+t-2 \\
t-1
\end{array}\right)\left(\begin{array}{c}
n-2 \\
i-2
\end{array}\right)+\sum_{k=i}^{n-1}\left(\begin{array}{c}
k+t-2 \\
t-1
\end{array}\right)\left(\begin{array}{c}
k-2 \\
i-2
\end{array}\right) \\
& =\sum_{k=i}^{n}\left(\begin{array}{c}
k+t-2 \\
t-1
\end{array}\right)\left(\begin{array}{c}
k-2 \\
i-2
\end{array}\right) .
\end{aligned}
$$

\section{Proof of the Main Theorem}

Throughout this section we let our matrices act on the right so that the cokernel of the map represented by a matrix is obtained by killing the row space.

Let $\mathbf{u}=u_{1}, \ldots, u_{n}$. Suppose we are given a Noetherian local ring $(R, m, k)$, an $R$-regular sequence $\mathbf{x}=x_{1}, \ldots, x_{n}$ and a $\mathbf{u}$-segment $T$. We will now proceed to construct a free resolution for $M$, the $R$-module associated with $T$ with respect to $(\mathbf{u}, \mathbf{x})$.

Let $d=\min _{f \in T} \operatorname{deg} f$ and $D=\max _{f \in T} \operatorname{deg} f$. Let $T_{\delta}=\{f \in T: \operatorname{deg} f=\delta\}$. Suppose there exists $d<\alpha<D$ such that $T_{\alpha}$ is empty. Let $S_{1}=\bigcup_{\delta<\alpha} T_{\delta}$ and $S_{2}=\bigcup_{\delta>\alpha} T_{\delta}$. Let $F_{i}$ be the submodule of $F$ generated by $w_{f}$ with $f \in S_{i}$ for $i=1,2$. Then $F=F_{1} \oplus F_{2}$. Observe that the each element in (0-1) is in either $F_{1}$ or $F_{2}$. Let $N_{i}$ be the submodule of $N$ generated by $N \cap F_{i}$ for $i=1,2$. Then $F / N \simeq F_{1} / N_{1} \oplus F_{2} / N_{2}$ and $F_{i} / N_{i}$ is the module associated with $S_{i}$ with respect to $(\mathbf{u}, \mathbf{x})$ for $i=1,2$. If $T$ is a $\mathbf{u}$-segment, then so are $S_{1}$ and $S_{2}$. Without loss of generality we will assume that $T_{\delta}$ is nonempty for $d \leq \delta \leq D$ to simplify notation. However, without this assumption the following argument is still valid with slight modification. Thus the results of Corollary 2.3, Theorem 0.2 and Corollary 0.3 still hold.

Order all the $T_{\delta}$. Write $\mathbf{x}=\left(x_{1}, \ldots, x_{n}\right)^{\text {tr }}$. For $d \leq \delta \leq D$ let

$$
\mathbf{X}_{\delta}=\left(\begin{array}{cccc}
\mathbf{x} & & & \\
& \mathbf{x} & & \\
& & \ddots & \\
& & & \mathbf{x}
\end{array}\right)^{n l_{\delta} \times l_{\delta}} .
$$

We can consider this matrix as having block entries of $n \times 1$ matrices with the row and column blocks indexed by the elements of $T_{\delta}$ in the given order. For $d \leq \delta \leq D-1$ let $\mathbf{R}_{\delta}=\left(\mathbf{r}_{f g}\right)_{\substack{f \in T_{\delta} \\ g \in T_{\delta+1}}}$ where $\mathbf{r}_{f g}=0$ if $f \nmid_{\mathbf{u}} g$ and $\mathbf{r}_{f g}=\mathbf{e}_{k}$ the column $n$-vector with 1 at the $k$-th position and 0 elsewhere if $g=u_{k} f$. The matrix $\mathbf{R}_{\delta}$ is an $n\left|T_{\delta}\right| \times\left|T_{\delta+1}\right|$ matrix. We can also consider $\mathbf{R}_{\delta}$ as a matrix having block entries of $n \times 1$ with the row blocks indexed by elements of $T_{\delta}$ in the given order and the column blocks indexed by $T_{\delta+1}$ in the given order. Now we can write down $M$ as the cokernel of

$$
\left(\begin{array}{ccccc}
\mathbf{X}_{d} & -\mathbf{R}_{d} & & & \\
& \mathbf{X}_{d+1} & -\mathbf{R}_{d+1} & & \\
& & \ddots & \ddots & \\
& & & \mathbf{X}_{D-1} & -\mathbf{R}_{D-1} \\
& & & & \mathbf{X}_{D}
\end{array}\right)
$$


We give a brief description of Koszul complexes: readers are referred to [16 for further details. If $\mathbf{x}=x_{1}, \ldots, x_{n}$ is any sequence of elements of a ring $R$, the Koszul complex $\mathcal{K}_{\bullet}(\mathbf{x} ; R)$ may be defined in the following fashion. One may identify $\mathcal{K}_{1}(\mathbf{x} ; R)$ with a free module $G$ on $n$ generators $u_{i}$, where the differential $d$ maps $u_{i}$ to $x_{i}$ in $\mathcal{K}_{0}(\mathbf{x} ; R)=R$, and then the entire complex may be identified with the exterior algebra $\wedge G$, where the map $d$ is extended to $\wedge G$ in the unique way that makes it a derivation of degree -1 (so that if $v \in \wedge^{i} G$ and $w \in \wedge^{j} G$ are homogeneous elements of $\wedge G$ of respective degrees $i$ and $j$, then $\left.d(u \wedge v)=(d u) \wedge v+(-1)^{i} u \wedge(d v)\right)$.

To be more specific, let $T_{i}$ be the set of the $i$-element subsets $\mathbf{t}$ of $\{1, \ldots, n\}$. Let $\langle\mathbf{t}\rangle$ be the increasing sequence $u_{t_{1}}<u_{t_{2}}<\cdots<u_{t_{i}}$ of elements of $\mathbf{t}$. Write $u_{\langle\mathbf{t}\rangle}$ for $u_{t_{1}} \wedge \cdots \wedge u_{t_{i}}$ and if $1 \leq j \leq i$, we write $\langle\mathbf{t}\rangle-j$ for this sequence with its $j$-th term omitted, a sequence of length $i-1$. With these conventions we have the explicit formula

$$
d_{i}\left(u_{\langle\mathbf{t}\rangle}\right)=\sum_{j=1}^{i}(-1)^{j-1} x_{t_{j}} u_{\langle\mathbf{t}\rangle-j} .
$$

Write $u_{\mathbf{t}}$ for $u_{\langle\mathbf{t}\rangle}$ for short. Order $T_{i}$ according to the lexicographical order of $\langle\mathbf{t}\rangle$. Denote by $\mathbf{A}_{i}^{n}\left(x_{1}, \ldots, x_{n}\right)$ the associated matrix of the differential map from $\mathcal{K}_{i}(\mathbf{x} ; R)$ to $\mathcal{K}_{i-1}(\mathbf{x} ; R)$ with respect to these bases. For instance, we have

$$
\mathbf{A}_{2}^{3}\left(x_{1}, x_{2}, x_{3}\right)=\left(\begin{array}{ccc}
-x_{2} & x_{1} & \\
-x_{3} & & x_{1} \\
& -x_{3} & x_{2}
\end{array}\right) .
$$

In this fashion, it is easy to check that

$$
\mathbf{A}_{i}^{n}\left(x_{1}, \ldots, x_{n}\right)=\left(\begin{array}{cc}
-\mathbf{A}_{i-1}^{n-1}\left(x_{2}, \ldots, x_{n}\right) & x_{1} \mathbf{I}_{\left(\begin{array}{c}
n-1 \\
i-1
\end{array}\right)} \\
\mathbf{0} & \mathbf{A}_{i}^{n-1}\left(x_{2}, \ldots, x_{n}\right)
\end{array}\right) .
$$

Proposition 2.1. Let $|T|=l$. Write

$$
\mathbf{X}_{\delta}^{i}=\left(\begin{array}{llll}
\mathbf{A}_{i}^{n}(\mathbf{x}) & & & \\
& \mathbf{A}_{i}^{n}(\mathbf{x}) & & \\
& & \ddots & \\
& & & \mathbf{A}_{i}^{n}(\mathbf{x})
\end{array}\right)_{\left(\begin{array}{c}
n \\
i
\end{array}\right)\left|T_{\delta}\right| \times\left(\begin{array}{c}
n \\
i-1
\end{array}\right)\left|T_{\delta}\right|}
$$

and

$$
\mathbf{R}_{\delta}^{i}=\left(\mathbf{A}_{i}^{n}\left(\mathbf{r}_{f g}\right)\right) \underset{\substack{f \in T_{\delta} \\ g \in T_{\delta+1}}}{ } .
$$

The following complex $K_{\bullet}$ is a free resolution of $M$ :

$$
0 \rightarrow R^{l\left(\begin{array}{l}
n \\
n
\end{array}\right)} \stackrel{A_{n}}{\longrightarrow} R^{l\left(\begin{array}{c}
n \\
n-1
\end{array}\right)} \stackrel{A_{n-1}}{\longrightarrow} \cdots \stackrel{A_{2}}{\longrightarrow} R^{l\left(\begin{array}{c}
n \\
1
\end{array}\right)} \stackrel{A_{1}}{\longrightarrow} R^{l\left(\begin{array}{l}
n \\
0
\end{array}\right)} \rightarrow M \rightarrow 0,
$$

where $A_{i}$ is the matrix

$$
\left(\begin{array}{ccccc}
\mathbf{X}_{d}^{i} & -\mathbf{R}_{d}^{i} & & & \\
& \mathbf{X}_{d+1}^{i} & -\mathbf{R}_{d+1}^{i} & & \\
& & \ddots & \ddots & \\
& & & \mathbf{X}_{D-1}^{i} & -\mathbf{R}_{D-1}^{i} \\
& & & & \mathbf{X}_{D}^{i}
\end{array}\right)
$$


Note that $A_{1}$ is the matrix $(2-1)$. The matrix $A_{i}$ may be considered as having block entries of size $\left(\begin{array}{c}n \\ i\end{array}\right) \times\left(\begin{array}{c}n \\ i-1\end{array}\right)$. The row and column blocks of $A_{i}$ are similarly indexed by the consecutive $T_{\delta}$. To be more precise, the row and column blocks of $\mathbf{X}_{\delta}$ are indexed by $T_{\delta}$ in the given order, the row blocks of $\mathbf{R}_{\delta}$ are indexed by $T_{\delta}$ and the column blocks are indexed by $T_{\delta+1}$ in the given order.

First we need to see that $K_{\bullet}$ is indeed a complex. That is, we need to see that $A_{i+1} A_{i}=0$ for $i=1,2, \ldots, n-1$. For this we need the following lemma.

Lemma 2.2. Let $\mathbf{X}=X_{1}, \ldots, X_{n}$ and $\mathbf{Y}=Y_{1}, \ldots, Y_{n}$ be two sets of indeterminates. Then

$$
\mathbf{A}_{i+1}^{n}(\mathbf{X}) \mathbf{A}_{i}^{n}(\mathbf{Y})+\mathbf{A}_{i+1}^{n}(\mathbf{Y}) \mathbf{A}_{i}^{n}(\mathbf{X})=\mathbf{0} .
$$

In particular, when $\mathbf{X}$ and $\mathbf{Y}$ are specialized by elements in a given ring this equation still holds.

Proof. Write $\mathbf{X}+\mathbf{Y}$ for the sequence $X_{1}+Y_{1}, \ldots, X_{n}+Y_{n}$. We have

$$
\begin{aligned}
\mathbf{0} & =\mathbf{A}_{i+1}^{n}(\mathbf{X}+\mathbf{Y}) \mathbf{A}_{i}^{n}(\mathbf{X}+\mathbf{Y}) \\
& =\left(\mathbf{A}_{i+1}^{n}(\mathbf{X})+\mathbf{A}_{i+1}^{n}(\mathbf{Y})\right)\left(\mathbf{A}_{i}^{n}(\mathbf{X})+\mathbf{A}_{i}^{n}(\mathbf{Y})\right) \\
& =\mathbf{A}_{i+1}^{n}(\mathbf{X}) \mathbf{A}_{i}^{n}(\mathbf{X})+\mathbf{A}_{i+1}^{n}(\mathbf{X}) \mathbf{A}_{i}^{n}(\mathbf{Y})+\mathbf{A}_{i+1}^{n}(\mathbf{Y}) \mathbf{A}_{i}^{n}(\mathbf{X})+\mathbf{A}_{+1}^{n} i(\mathbf{Y}) \mathbf{A}_{i}^{n}(\mathbf{Y}) \\
& =\mathbf{A}_{i+1}^{n}(\mathbf{X}) \mathbf{A}_{i}^{n}(\mathbf{Y})+\mathbf{A}_{i+1}^{n}(\mathbf{Y}) \mathbf{A}_{i}^{n}(\mathbf{X})
\end{aligned}
$$

Proof of Proposition 2.1. For $1 \leq i \leq n-1$ the product $A_{i+1} A_{i}$ equals

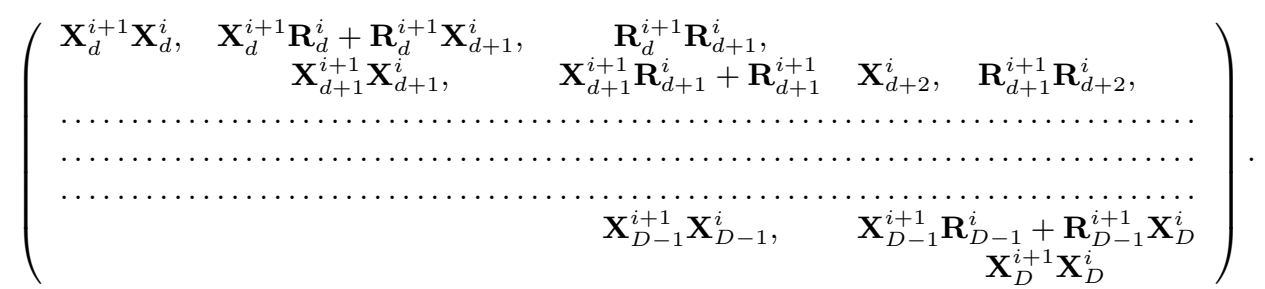

We need to check that

(1) $\mathbf{X}_{\delta}^{i+1} \mathbf{X}_{\delta}^{i}=\mathbf{0}$ for $d \leq \delta \leq D$;

(2) $\mathbf{X}_{\delta}^{i+1} \mathbf{R}_{\delta}^{i}+\mathbf{R}_{\delta}^{i+1} \mathbf{X}_{\delta+1}^{i}=\mathbf{0}$ for $d \leq \delta \leq D-1$; and

(3) $\mathbf{R}_{\delta}^{i+1} \mathbf{R}_{\delta+1}^{i}=\mathbf{0}$ for $d \leq \delta \leq D-1$.

It is quite easy to check (1). To check (2), observe that the matrix $\mathbf{X}_{\delta}^{i+1} \mathbf{R}_{\delta}^{i}+$ $\mathbf{R}_{\delta}^{i+1} \mathbf{X}_{\delta+1}^{i}$ has its row blocks indexed by $T_{\delta}$ and column blocks indexed by $T_{\delta+1}$. Take $f \in T_{\delta}$ and $g \in T_{\delta+1}$. The $(f, g)$-block entry of $\mathbf{X}_{\delta}^{i+1} \mathbf{R}_{\delta}^{i}+\mathbf{R}_{\delta}^{i+1} \mathbf{X}_{\delta+1}^{i}$ is

$$
\mathbf{A}_{i+1}^{n}(\mathbf{x}) \mathbf{A}_{i}^{n}\left(\mathbf{r}_{f g}\right)+\mathbf{A}_{i+1}^{n}\left(\mathbf{r}_{f g}\right) \mathbf{A}_{i}^{n}(\mathbf{x})=\mathbf{0}
$$

by Lemma 2.2. To check (3) note that the matrix $\mathbf{R}_{\delta}^{i+1} \mathbf{R}_{\delta+1}^{i}$ has its row blocks indexed by $T_{\delta}$ and column blocks indexed by $T_{\delta+2}$. Take $f \in T_{\delta}$ and $g \in T_{\delta+2}$. The $(f, g)$-block entry of $\mathbf{R}_{\delta}^{i+1} \mathbf{R}_{\delta+1}^{i}$ is

$$
\sum_{h \in T_{\delta+1}} \mathbf{A}_{i+1}^{n}\left(\mathbf{r}_{f h}\right) \mathbf{A}_{i}^{n}\left(\mathbf{r}_{h g}\right) .
$$


If $f \nmid_{\mathbf{u}} g$, then either $\mathbf{r}_{f h}$ or $\mathbf{r}_{h g}$ is $\mathbf{0}$ for each $h \in T_{\delta+1}$. If $g=u_{j} u_{k} f$, then the matrix $(2-3)$ equals

$$
\begin{aligned}
& \mathbf{A}_{i+1}^{n}\left(\mathbf{r}_{f, u_{j} f}\right) \mathbf{A}_{i}^{n}\left(\mathbf{r}_{u_{j} f, g}\right)+\mathbf{A}_{i+1}^{n}\left(\mathbf{r}_{f, u_{k} f}\right) \mathbf{A}_{i}^{n}\left(\mathbf{r}_{u_{k} f, g}\right) \\
= & \mathbf{A}_{i+1}^{n}\left(\mathbf{e}_{j}\right) \mathbf{A}_{i}^{n}\left(\mathbf{e}_{k}\right)+\mathbf{A}_{i+1}^{n}\left(\mathbf{e}_{k}\right) \mathbf{A}_{i}^{n}\left(\mathbf{e}_{j}\right)=\mathbf{0}
\end{aligned}
$$

by Lemma 2.2 again. Thus we have proved $K_{\bullet}$ is indeed a complex. Next we need to show that $K$. is acyclic.

Remember that the rank of $\mathbf{A}_{i}^{n}(\mathbf{x})$ is $\left(\begin{array}{c}n-1 \\ i-1\end{array}\right)$ and the rank ideal of $\mathbf{A}_{i}^{n}(\mathbf{x})$ contains $x_{k}^{\left(\begin{array}{c}n-1 \\ i-1\end{array}\right)}$ for each $k$. Observe the position of $\mathbf{A}_{i}^{n}(\mathbf{x})$ in the matrix (2-2). We can see that the rank of $A_{i}$ is at least $\left(\begin{array}{c}n-1 \\ i-1\end{array}\right) l$. The entries of the $A_{i}$ 's involve only elements like $\pm x_{k}, \pm 1$, and 0 . If we assume $x_{1}, \ldots, x_{n}$ are distinct indeterminates, then we can think of the $A_{i}$ as matrices with entries inside $\mathbb{Z}\left[x_{1} \ldots, x_{n}\right]$, which is a domain. Since in this case we still have $A_{i+1} A_{i}=0$, we should have $\operatorname{rk} A_{i}+\operatorname{rk} A_{i+1} \leq$ $\left(\begin{array}{l}n \\ i\end{array}\right) l$. Since the rank of $A_{i}$ cannot increase if $\mathbf{x}$ are specialized by any sequence of elements in $R$, we have $\operatorname{rk} A_{i}=\left(\begin{array}{c}n-1 \\ i-1\end{array}\right) l$ even if we drop the condition $\mathbf{x}$ are distinct indeterminants. It is obvious the rank ideal of $A_{i}$ contains $x_{k}^{\left(\begin{array}{c}n-1 \\ i-1\end{array}\right) l}$ for each $k$. Thus the depth of the rank ideal of $A_{i}=n \geq i$ for $1 \leq i \leq n$. By the Eisenbud-Buchsbaum acyclicity criterion the complex $K_{\bullet}$ is acyclic.

We can now proceed to compute the Betti numbers of $M$. In fact we have the following corollary.

Corollary 2.3. With the same notation as in Proposition 2.1 we have

$$
\left\{\begin{array}{l}
r_{i}(M)=\left(\begin{array}{c}
n-1 \\
i-1
\end{array}\right) l-\sum_{\delta=d}^{D-1} \mathrm{rk} \mathbf{R}_{\delta}^{i} \\
\beta_{i}(M)=\left(\begin{array}{c}
n \\
i
\end{array}\right) l-\sum_{\delta=d}^{D-1} \mathrm{rk} \mathbf{R}_{\delta}^{i}-\sum_{\delta=d}^{D-1} \mathrm{rk} \mathbf{R}_{\delta}^{i+1}
\end{array}\right.
$$

for all $i$.

Proof. This follows from the fact that $r_{i}(M)$ is equal to the rank of the matrix in a minimal free resolution for $M$. The complex $K_{\bullet}$ is the direct product of a minimal free resolution for $M$ and a split exact sequence. Hence if we use $\overline{A_{i}}$ to denote the image of $A_{i}$ modulo $m$, we have

$$
\begin{aligned}
& r_{i}(M)=\operatorname{rk} A_{i}-\operatorname{rk} \overline{A_{i}} \\
& =l\left(\begin{array}{c}
n-1 \\
i-1
\end{array}\right)-\mathrm{rk}\left(\begin{array}{ccccc}
\mathbf{0} & -\mathbf{R}_{d}^{i} & & & \\
& \mathbf{0} & -\mathbf{R}_{d+1}^{i} & & \\
& & \ddots & \ddots & \\
& & & \mathbf{0} & -\mathbf{R}_{D-1}^{i} \\
& & & & \mathbf{0}
\end{array}\right) .
\end{aligned}
$$

Thus the result follows.

To compute the rank of $\mathbf{R}_{\delta}^{i}$ directly for specific examples is usually not as difficult as it looks. However, we have a better solution.

Definition 2.4. Write $\mathbf{r}_{f g}=\left(r_{f g}^{1}, r_{f g}^{2}, \ldots, r_{f g}^{n}\right)^{\text {tr }}$. Let $\mathbf{r}_{\delta}^{k}=\left(r_{f g}^{k}\right)_{\substack{f \in T_{\delta+1} \\ g \in T_{\delta}}}$. Note that the matrix $\mathbf{r}_{\delta}^{k}$ describes the action of multiplying by $x_{k}$ which sends elements of 
degree $\delta$ to elements of degree $\delta+1$. We will call $\left\{\mathbf{r}_{\delta}^{k}\right\}_{k, \delta}$ the structure matrices associated with $T$.

In 4 it is shown that

$$
\operatorname{rk} \mathbf{R}_{\delta}^{i}=\operatorname{rk}_{i}^{n}\left(\mathbf{r}_{\delta}^{1}, \ldots, \mathbf{r}_{\delta}^{n}\right) .
$$

In fact $\mathbf{A}_{i}^{n}\left(\mathbf{r}_{\delta}^{1}, \ldots, \mathbf{r}_{\delta}^{n}\right)$ can be achieved by permuting rows and columns of $\mathbf{R}_{\delta}^{i}$. Reorder $T_{\delta}$ and $T_{\delta+1}$ if necessary so that $\mathbf{r}_{\delta}^{1}=\left(\begin{array}{ll}\mathbf{I} & \mathbf{0} \\ \mathbf{0} & \mathbf{0}\end{array}\right)$. Let $T_{\delta}^{\prime}=\left\{f \in T_{\delta}\right.$ : the $f$-row in $\mathbf{r}_{\delta}^{1}$ is $\left.\mathbf{0}\right\}$ and let $T_{\delta+1}^{\prime \prime}=\left\{f \in T_{\delta}\right.$ : the $g$-column in $\mathbf{r}_{\delta}^{1}$ is $\left.\mathbf{0}\right\}$. In other words, $T_{\delta}^{\prime}=\left\{f \in T_{\delta}: u_{1} f \notin T_{\delta+1}\right\}$ and $T_{\delta+1}^{\prime \prime}=\left\{g \in T_{\delta+1}: g \notin u_{1} T_{\delta}\right\}$. Write $\mathbf{r}_{\delta}^{k}=\left(\begin{array}{c}\mathfrak{s}_{\delta}^{k} \\ \mathbf{s}_{\delta}^{k}\end{array}\right)$ where $\mathfrak{s}_{\delta}^{k}=\left(r_{f g}^{k}\right)_{\substack{f \in T_{\delta} \backslash T_{\delta}^{\prime} \\ g \in T_{\delta+1}}}$ and $\mathbf{s}_{\delta}^{k}=\left(r_{f g}^{k}\right)_{\substack{f \in T_{\delta+1}^{\prime} \\ g \in T_{\delta}^{\prime}}}$ and $\mathbf{r}_{\delta}^{k}=\left(\begin{array}{ll}\mathfrak{t}_{\delta}^{k} & \overline{\mathbf{t}_{\delta}^{k}}\end{array}\right)$ where $\mathfrak{t}_{\delta}^{k}=\left(r_{f g}^{k}\right) \underset{g \in T_{\delta+1} \backslash T_{\delta+1}^{\prime \prime}}{f \in T_{\delta}}$ and $\overline{\mathbf{t}_{\delta}^{k}}=\left(r_{f g}^{k}\right)_{g \in T_{\delta+1}^{\prime \prime}}^{f \in T_{\delta}}$. By appropriately exchanging columns and rows the matrix $\mathbf{A}_{i}^{n}\left(\mathbf{r}_{\delta}^{1}, \ldots, \mathbf{r}_{\delta}^{n}\right)$ becomes

$$
\left(\begin{array}{ccc}
-\mathbf{A}_{i-1}^{n-1}\left(\mathfrak{s}_{\delta}^{2}, \ldots, \mathfrak{s}_{\delta}^{n}\right) & \mathbf{I} & \mathbf{0} \\
-\mathbf{A}_{i-1}^{n-1}\left(\mathbf{s}_{\delta}^{2}, \ldots, \mathbf{s}_{\delta}^{n}\right) & \mathbf{0} & \mathbf{0} \\
\mathbf{0} & \mathbf{A}_{i}^{n-1}\left(\mathfrak{t}_{\delta}^{2}, \ldots, \mathfrak{t}_{\delta}^{n}\right) & \mathbf{A}_{i}^{n-1}\left(\overline{\mathbf{t}_{\delta}^{2}}, \ldots, \mathbf{t}_{\delta}^{n}\right)
\end{array}\right) .
$$

By doing row and column operations the matrix becomes

$$
\left(\begin{array}{ccc}
\mathbf{0} & \mathbf{I} & \mathbf{0} \\
-\mathbf{A}_{i-1}^{n-1}\left(\overline{\mathbf{s}_{\delta}^{2}}, \ldots, \mathbf{s}_{\delta}^{n}\right) & \mathbf{0} & \mathbf{0} \\
\mathbf{A}_{i}^{n-1}\left(\mathfrak{t}_{\delta}^{2}, \ldots, \mathbf{t}_{\delta}^{n}\right) \mathbf{A}_{i-1}^{n-1}\left(\mathfrak{s}_{\delta}^{2}, \ldots, \mathfrak{s}_{\delta}^{n}\right) & \mathbf{0} & \mathbf{A}_{i}^{n-1}\left(\overline{\mathbf{t}_{\delta}^{2}}, \ldots, \overline{\mathbf{t}}_{\delta}^{n}\right)
\end{array}\right) .
$$

Lemma 2.5. The matrix block $\mathbf{A}_{i}^{n-1}\left(\mathfrak{t}_{\delta}^{2}, \ldots, \mathfrak{t}_{\delta}^{n}\right) \mathbf{A}_{i-1}^{n-1}\left(\mathfrak{s}_{\delta}^{2}, \ldots, \mathfrak{s}_{\delta}^{n}\right)$ inside the matrix above is the zero matrix.

Proof. This lemma is true if we can show that $\mathfrak{t}_{\delta}^{j} \mathfrak{s}_{\delta}^{k}=\mathfrak{t}_{\delta}^{k} \mathfrak{s}_{\delta}^{j}$. For $j, k \neq 1$ note that the entries of $\mathfrak{t}_{\delta}^{j}$ are indexed by $T_{\delta}, T_{\delta+1} \backslash T_{\delta+1}^{\prime \prime}$ and the entries of $\mathfrak{s}_{\delta}^{k}$ are indexed by $T_{\delta} \backslash T_{\delta}^{\prime}$ and $T_{\delta+1}$. The elements of $T_{\delta} \backslash T_{\delta}^{\prime}$ correspond bijectively with $T_{\delta+1} \backslash T_{\delta+1}^{\prime \prime}$ by sending $h$ to $u_{1} h$. Take $f \in T_{\delta}$ and $g \in T_{\delta+1}$. The $(f, g)$-entry of $\mathfrak{t}_{\delta}^{j} \mathfrak{s}_{\delta}^{k}$ is

$$
\sum_{h \in T_{\delta} \backslash T_{\delta}^{\prime}} r_{f, u_{1} h}^{j} r_{h, g}^{k}= \begin{cases}1, & \text { if } u_{j} u_{k} f=u_{1} g \\ 0, & \text { otherwise. }\end{cases}
$$

Similarly, we can see that the $(f, g)$-entry of $\mathfrak{t}_{\delta}^{k} \mathfrak{s}_{\delta}^{j}$ is 1 if $u_{j} u_{k} f=u_{1} g$ and is 0 otherwise. It is not surprising this lemma should hold since both $\mathfrak{t}_{\delta}^{j} \mathfrak{s}_{\delta}^{k}$ and $\mathfrak{t}_{\delta}^{k} \mathfrak{s}_{\delta}^{j}$ describe the action multiplying by $x_{j} x_{k}$ on those $w_{f}, f \in T_{\delta}$, not killed by $x_{1}$.

Let $l_{\delta}=\left|T_{\delta} \backslash T_{\delta}^{\prime}\right|=\left|T_{\delta+1} \backslash T_{\delta+1}^{\prime \prime}\right|$. Now by Lemma 2.5 we have

$$
\operatorname{rk} \mathbf{R}_{\delta}^{i}=l_{\delta}\left(\begin{array}{c}
n-1 \\
i-1
\end{array}\right)+\operatorname{rk} \mathbf{A}_{i-1}^{n-1}\left(\overline{\mathbf{s}_{\delta}^{2}}, \ldots, \overline{\mathbf{s}_{\delta}^{n}}\right)+\operatorname{rk} \mathbf{A}_{i}^{n-1}\left(\overline{\mathbf{t}_{\delta}^{2}}, \ldots, \mathbf{t}_{\delta}^{n}\right) .
$$

Note that $T^{\prime}=\bigcup_{\delta} T_{\delta}^{\prime}$ and $T^{\prime \prime}=\bigcup_{\delta} T_{\delta}^{\prime \prime}$.

Lemma 2.6. Let $\overline{\mathbf{u}}=u_{2}, \ldots, u_{n}$. Then $T^{\prime}$ and $T^{\prime \prime}$ are both $\overline{\mathbf{u}}$-segments.

Proof. To show $T^{\prime}$ is a $\overline{\mathbf{u}}$-segment, take $f, h \in T^{\prime}$ and let $\left.f\right|_{\overline{\mathbf{u}}} g$ and $\left.g\right|_{\overline{\mathbf{u}}} h$. Since $T$ is a $\mathbf{u}$-segment, we have $g \in T$. If $u_{1} g \in T$, then $\left.\left.f\right|_{\mathbf{u}} u_{1} f\right|_{\mathbf{u}} u_{1} g$ implies $u_{1} f \in T$; this is a contradiction. Hence $g \in T^{\prime}$. 
To show $T^{\prime \prime}$ is a $\overline{\mathbf{u}}$-segment, let $\left.\left.f\right|_{\overline{\mathbf{u}}} g\right|_{\overline{\mathbf{u}}} h$ for some $f, h \in T^{\prime \prime}$. Again $g \in T$ since $T$ is a $\mathbf{u}$-segment. Suppose $g=u_{1} g^{\prime}$ for some $g^{\prime} \in T$. Then $u_{1} \mid h$. Suppose $h=u_{1} h^{\prime}$. Then $\left.\left.g^{\prime}\right|_{\mathbf{u}} h^{\prime}\right|_{\mathbf{u}} h$. Hence $h^{\prime} \in T$. Thus $h \notin T^{\prime \prime}$, a contradiction.

We will round up the proof of Theorem 0.2 now.

Proof of Theorem 0.2. Let $f \in T_{\delta}^{\prime}$ and $2 \leq k \leq n$. If $u_{k} f \in T_{\delta+1}$, then we claim that $u_{k} f \in T_{\delta+1}^{\prime}$. To see this we need to check that $u_{1} u_{k} f \notin T_{\delta+2}$. If $u_{1} u_{k} f \in T_{\delta+2}$, then $\left.\left.f\right|_{\mathbf{u}} u_{1} f\right|_{\mathbf{u}} u_{1} u_{k} f$ implies that $u_{1} f \in T$ and hence $f \notin T_{\delta}^{\prime}$, a contradiction. Thus we can write $\overline{\mathbf{s}_{\delta}^{k}}=\left(\begin{array}{ll}\mathbf{0} & \mathbf{s}_{\delta}^{k}\end{array}\right)$, where $\mathbf{s}_{\delta}^{k}=\left(r_{f g}^{k}\right)_{g \in T_{\delta}^{\prime}}$. If $f \in T_{\delta} \backslash T_{\delta}^{\prime \prime}$ and $2 \leq k \leq n$, then $f=u_{1} h$ for some $h \in T_{\delta-1}$. If $u_{k} f \in T_{\delta+1}$, then $u_{k} h \in T$ since $\left.\left.h\right|_{\mathbf{u}} u_{k} h\right|_{\mathbf{u}} u_{k} f$. Hence $u_{k} f \in T_{\delta+1} \backslash T_{\delta+1}^{\prime \prime}$. Thus we can write $\mathbf{t}_{\delta}^{\bar{k}}=\left(\begin{array}{c}\mathbf{0} \\ \mathbf{t}_{\delta}^{k}\end{array}\right)$ where $\mathbf{t}_{\delta}^{k}=\left(r_{f g}^{k}\right)_{\substack{f \in T_{\delta}^{\prime \prime} \\ g \in T_{\delta+1}^{\prime \prime}}}$. By permuting columns the matrix $\mathbf{A}_{i}^{n}\left(\overline{\mathbf{s}}_{\delta}^{2}, \ldots, \mathbf{s}_{\delta}^{n}\right)$ becomes $\left(\mathbf{0} \quad \mathbf{A}_{i}^{n}\left(\mathbf{s}_{\delta}^{2}, \ldots, \mathbf{s}_{\delta}^{n}\right)\right)$. By permuting rows the matrix $\mathbf{A}_{i}^{n}\left(\overline{\mathbf{t}}_{\delta}^{2}, \ldots, \overline{\mathbf{t}}_{\delta}^{n}\right)$ becomes $\left(\begin{array}{c}\mathbf{0} \\ \mathbf{A}_{i}^{n}\left(\mathbf{t}_{\delta}^{2}, \ldots, \mathbf{t}_{\delta}^{n}\right)\end{array}\right)$.

Now let $\overline{\mathbf{x}}=x_{2}, \ldots, x_{n}$ and let $\overline{\mathbf{u}}=u_{2}, \ldots, u_{n}$. Let $M^{\prime}$ be the $R$-module associated with $T^{\prime}$ with respect to $(\overline{\mathbf{u}}, \overline{\mathbf{x}})$ and let $M^{\prime \prime}$ be the $R$-module associated with $T^{\prime \prime}$ with respect to $(\overline{\mathbf{u}}, \overline{\mathbf{x}})$. It is easy to see that $\left\{\mathbf{s}_{\delta}^{k}\right\}_{k, \delta}$ are the structure matrices of $T^{\prime}$ while $\left\{\mathbf{t}_{\delta}^{k}\right\}_{k, \delta}$ are the structure matrices of $T^{\prime \prime}$.

Let $l^{\prime}=\left|T^{\prime}\right|=\left|T^{\prime \prime}\right|=l-\sum_{d}^{D} l_{\delta}$. Then by induction on $n$ and by $(2-4)$ we have

$$
\begin{aligned}
& r_{i}(M)=\left(\begin{array}{c}
n-1 \\
i-1
\end{array}\right) l-\sum_{\delta=d}^{D-1} \operatorname{rk} \mathbf{A}_{i}^{n}\left(\mathbf{r}_{\delta}^{1}, \ldots, \mathbf{r}_{\delta}^{n}\right) \\
= & \left(\begin{array}{c}
n-1 \\
i-1
\end{array}\right) l-\sum_{\delta=d}^{D-1}\left[l_{\delta}\left(\begin{array}{c}
n-1 \\
i-1
\end{array}\right)+\operatorname{rk} \mathbf{A}_{i-1}^{n-1}\left(\mathbf{s}_{\delta}^{2}, \ldots, \mathbf{s}_{\delta}^{n}\right)+\operatorname{rk~}_{i}^{n-1}\left(\mathbf{t}_{\delta}^{2}, \ldots, \mathbf{t}_{\delta}^{n}\right)\right] \\
= & \left(\begin{array}{c}
n-2 \\
i-2
\end{array}\right) l^{\prime}-\sum_{\delta=d}^{D-1}\left[\operatorname{rk} \mathbf{A}_{i-1}^{n-1}\left(\mathbf{s}_{\delta}^{2}, \ldots, \mathbf{s}_{\delta}^{n}\right)\right]+\left(\begin{array}{c}
n-2 \\
i-1
\end{array}\right) l^{\prime}-\sum_{\delta=d}^{D-1}\left[\operatorname{rk} \mathbf{A}_{i}^{n-1}\left(\mathbf{t}_{\delta}^{2}, \ldots, \mathbf{t}_{\delta}^{n}\right)\right] \\
= & r_{i-1}\left(M^{\prime}\right)+r_{i}\left(M^{\prime \prime}\right) .
\end{aligned}
$$

This completes the proof of Theorem 0.2.

Proof of Corollary 0.3. Suppose $M$ is of essentially monomial type with respect to the $R$-regular sequence $\mathbf{x}=x_{1}, \ldots, x_{n}$. Let $\mathbf{u}=u_{1}, \ldots, u_{n}$. Find a $\mathbf{u}$-segment $T$ such that $M$ is isomorphic to the module associated with $T$ with respect to $(\mathbf{u}, \mathbf{x})$. We will use the same notation as in Corollary 2.3. When $i=1$ we have

$$
r_{1}(M)=l-\sum_{d}^{D-1} \operatorname{rk} R_{\delta} \geq l-\sum_{d+1}^{D}\left|T_{\delta}\right|=\left|T_{d}\right| \geq 1
$$

Now by Theorem 0.2 and by induction on $n$ we have

$$
r_{i}(M)=r_{i-1}\left(M^{\prime}\right)+r_{i}\left(M^{\prime \prime}\right) \geq\left(\begin{array}{c}
n-2 \\
i-2
\end{array}\right)+\left(\begin{array}{c}
n-2 \\
i-1
\end{array}\right)=\left(\begin{array}{c}
n-1 \\
i-1
\end{array}\right) .
$$

Furthermore, we have

$$
\beta_{i}(M)=r_{i}(M)+r_{i+1}(M) \geq\left(\begin{array}{l}
n \\
i
\end{array}\right)
$$




\section{REFERENCES}

1. D. Buchsbaum and D. Eisenbud, Algebra structures for finite free resolutions, and some structure theorems for ideals of codimension 3, Amer. J. Math. 99 (3) (1977), 447-485. MR 56:11983

2. - Generic free resolutions and a family of generically perfect fields, Adv. in Math. 18 (1975), 245-301. MR 53:391

3. What makes a complex exact, J. of Algebra (1973), 259-268. MR 47:3369

4. S.-T. Chang, Betti numbers of modules of exponent two over regular local rings, J. of Algebra 193 (1997), 640-659. CMP 97:15

5. H. Charalambous, Lower bounds for betti numbers of multigarded modules, J. of Algebra 137 (1991), 491-500. MR 92b:13020

6. H. Charalambous and E. G. Evans, Problems on Betti Numbers of finite length modules, Free resolutions in Commutative Algebra and Algebraic Geometry (D. Eisenbud and C. Huneke, eds.), Research Notes in Mathematics, vol. 2, Jones and Bartlett, Boston, 1992, pp. 25-33. MR 93e: 13018

7. H. Charalambous, E. G. Evans and M. Miller, Betti numbers for modules of finite legnth, Proc. of the A. M. S. 109 (1990), 63-70. MR 90j:13021

8. D. Dugger, Betti numbers of almost complete intersections, preprint.

9. E. G. Evans and P. Griffith, Syzygies, London Math. Soc. Lecture Note Series, Cambridge University Press, 1985. MR 87b:13001

10. 133 (1988), 267-276. MR 89d:13014

11. R. Hartshorne, Algebraic vector bundles on projective spaces: a problem list, Topology $\mathbf{1 8}$ (1979), 117-128. MR 81m:14014

12. J. Herzog and M. Kühl, On the Betti numbers of finite pure and linear resolutions, Comm. Algebra 12 13-14 (1984), 1627-1646. MR 85e:13021

13. G. Horrocks, Vector bundles on the punctured spectrum of a regular local ring, Proc. London Math. Soc. (3) 14 (1964), 689-713. MR 30:120

14. C. Huneke and B. Ulrich, The structure of linkage, Annals of Math. 126 (1987), 277-334. MR 88k:13020

15. L. Santoni, Horrocks' question for monomially graded modules, Pacific J. of Math. 141, (1) (1990), 105-124. MR 91b:13022

16. J.-P. Serre, Algèbre locale. Multiplicités, Lecture Notes in Mathematics, Springer-Verlag, Berlin-Heidelberg-New York, 1965. MR 34:1352

Department of Mathematics, National Chung Cheng University, Minghsiung, Chiayi 621, TAIWAN, R.O.C.

E-mail address: stchang@math.ccu.edu.tw 